\title{
INFERENCES ABOUT A LINEAR COMBINATION OF PROPORTIONS
}

\author{
A. Martín Andrés ${ }^{*}(1)$, M. Álvarez Hernández ${ }^{(1)}$ and I. Herranz Tejedor ${ }^{(2)}$ \\ ${ }^{(1)}$ Bioestadística. Facultad de Medicina. Universidad de Granada. 18071 Granada. Spain. \\ ${ }^{(2)}$ Bioestadística. Facultad de Medicina. Universidad Complutense de Madrid. 28040 Madrid. Spain.
}

\section{SUMMARY}

Statistical methods for carrying out asymptotic inferences (tests or confidence intervals) relative to one or two independent binomial proportions are very frequent. However inferences about a linear combination of $K$ independent proportions $L=\Sigma \beta_{i} p_{i}$ (in which the first two are special cases) have had very little attention paid to them (focused exclusively on the classic Wald method). In this paper the authors approach the problem from the more efficient viewpoint of the score method, which can be solved using a free program which is available from the webpage quoted in the article. In addition the paper offers approximate formulas that are easy to calculate, gives a general proof of Agresti's heuristic method (consisting of adding a certain number of successes and failures to the original results before applying Wald's method) and, finally, it proves that the score method (which verifies the desirable properties of spatial and parametric convexity) is the best option in comparison with other methods.

KEY WORDS: Agresti method; binomial distribution; confidence interval; linear function of proportions; score method; Wald method.

\section{Introduction.}

Asymptotic inferences relative to binomial proportions are very usual in applied research, and this has resulted in a large number of statisticians developing appropriate theoretical procedures. In particular, the case of one or two independent proportions has received a great deal of attention in recent years. For example, in 2008 alone thirteen articles

\footnotetext{
${ }^{*}$ Correspondence to: Bioestadística. Facultad de Medicina. Universidad de Granada. 18071 Granada. Spain. Email: amartina@ugr.es. Phone:34-58-244080. Fax: 34-58-246117.
} 
about the difference, ratio or odds-ratio of two independent proportions have been published $^{1-13}$.

Surprisingly, however, the asymptotic inferences concerning a linear combination $\left(L=\Sigma \beta_{i} p_{i}\right)$ with $K>2$ independent binomial proportions have received very little attention, despite their great practical importance (e.g., dose-response studies, public-health surveys, multicenter clinical trials, agricultural experiments, etc. ${ }^{14}$. Even more surprisingly, up till now the problem has been approached only from the points of view of the confidence intervals obtained using Wald's method.

In this paper, the problem is dealt with from the point of view of the hypothesis tests, and the confidence interval $(\mathrm{CI})$ is obtained by inverting the test. Moreover, the problem is resolved by using the score method, which, by general agreement, produces better results that Wald's method in cases $K=1^{15}, K=2^{16}$ and in general for any parameter of a contingency table ${ }^{5}$. Finally, the paper offers a theoretical proof of the heuristic result that Wald's 95\% confidence interval improves if $2 / K$ successes and failures are added to each sample -Agresti and Coull ${ }^{15}$ for $K=1$, Agresti and $\mathrm{Caffo}^{17}$ for $K=2$ and Price and Bonett ${ }^{18}$ for $K>2-$, at the same time as it generalizes the result for any confidence value.

\section{Examples.}

Price and Bonett ${ }^{18}$ refer to a study by Cohen et al. ${ }^{19}$ in which 120 rats were randomly assigned to four diets (high or low fat and with fiber or without fiber). The absence or presence of a tumor was recorded for each rat. Table 1 shows the data and the contrasts $L$ of interest $\left(L_{2}\right.$ for evaluating the effect of dietary fiber; $L_{3}$ for evaluating the effect of the level of fat; $L_{1}$ for evaluating the interaction between $L_{1}$ and $L_{2}$, that is, the difference between the effects of fiber according to which one or other of the fat levels are determined). In all cases $\Sigma \beta_{i}=0$.

Tebbs and Roths ${ }^{14}$ refer to the data (Table 2) in a multicenter clinical trial where the aim was to evaluate the efficacy of a reduced-salt regime in treating male infants for acute watery diarrhea. One of the characteristics measured was the number of infants experiencing fever at 
admission or during the trial. The aim is to estimate the pooled proportion of subjects who respond to treatment. Because the level of participation is likely different depending on the location, a natural estimate of the pooled proportion is the average of the response probabilities from the $K=6$ sites, i.e. $L=\Sigma \beta_{i} p_{i}$ with $\beta_{i}=n_{i} / \Sigma n_{i}$. Now $\Sigma \beta_{i} \neq 0$. A similar problem often arises in the metaanalysis, where it is common to take linear combinations across studies.

\section{The Wald method and the adjusted Wald method.}

Let $K$ be independent binomial random variables $x_{i} \sim B\left(n_{i} ; p_{i}\right)$, where $\mathrm{i}=1,2, \ldots, K$ and let $L=\Sigma \beta_{i} p_{i}$ the parameter of interest (where the proportions $p_{i}$ are unknown and the parameters $\beta_{i} \neq 0$ known). When $K=1$ and $\beta_{1}=1$, the parameter of interest is the simple proportion $p_{1}$. When $K=2, \beta_{1}=1$ and $\beta_{2}=+1$, the parameter of interest is the difference between two proportions $d=p_{2}-p_{1}$. Generally speaking, the parameter of interest $L$ may refer to a contrast (if $\Sigma \beta_{i}=0$ ) or to a more general combination (if $\Sigma \beta_{i} \neq 0$ ).

Whatever the situation the statistic $\bar{L}=\sum \beta_{i} \bar{p}_{i}$, where $\bar{p}_{i}=x_{i} / n_{i}$, is asymptotically normal with mean $L=\Sigma \beta_{i} p_{i}$ and variance $V=\sum \beta_{i}^{2} p_{i} q_{i} / n_{i}$, where $q_{i}=1-p_{i}$. As a result, the test for contrasting $H_{0}: L=\lambda$ vs. $H_{l}: L \neq \lambda$ will be based on the statistic $z=(\bar{L}-\lambda) / \sqrt{V}$, which must be compared in traditional fashion with $\mathrm{z}_{\alpha / 2}$ (the $\alpha / 2$-upper percentile of the typical normal distribution). Inverting the test - that is, making $z^{2}=z_{\alpha / 2}^{2}$ and working out $\lambda-\mathrm{a}(1-\alpha)$ CI for $L$ is obtained: $\bar{L} \pm z_{\alpha / 2} \sqrt{V}$. As the values of $p_{i}$ are unknown, the simplest option is to substitute $p_{i}$ by $\bar{p}_{i}$, which yields to the following Wald's statistic and Wald's CI (where $\left.\bar{q}_{i}=1-\bar{p}_{i}\right)$

$$
z_{1}=(\bar{L}-\lambda) / \sqrt{V_{1}}, \quad C I_{1}: \bar{L} \pm z_{\alpha / 2} \sqrt{V_{1}} \text { where } V_{1}=\sum \beta_{i}^{2} \bar{p}_{i} \bar{q}_{i} / n_{i} .
$$

Price and Bonett ${ }^{18}$ found heuristically that Wald's CI improves substantially if expression (1) is obtained not based on the original $x_{i}$ and $n_{i}$, but on $x_{i}+2 / K$ and $n_{i}+4 / K$, that is if $2 / K$ 
successes and $2 / K$ failures are added to the original data. This yields the adjusted Wald method $\mathrm{W}(+2 / K)$, in contrast to the original Wald method $\mathrm{W}(+0)$, which is also applicable in the case of the test. The procedure is compatible with the one recommended by Agresti and Coull ${ }^{15}$ for the case of one proportion $\left(K=1, \beta_{1}=1\right)$ and by Agresti and $\mathrm{Caffo}^{17}$ for the case of the difference between the two proportions $\left(K=2, \beta_{1}=-1, \beta_{2}=+1\right)$. The origin of the method is to be found in the case of one proportion. Agresti and Coull ${ }^{15}$ proved that Wilson's CI (which proceeds from the score test) has a midpoint that is equal to that of method $\mathrm{W}\left(+z_{\alpha / 2}^{2} / 2\right)$, thus yielding a theoretical justification concerning the good behavior of method $\mathrm{W}(+2)$ in the case of one proportion, because $z_{2.5 \%}^{2}=1.96^{2} \simeq 4$. The natural extension of this to case $K>1$ is $\mathrm{W}\left(+z_{\alpha / 2}^{2} / 2 K\right) \simeq \mathrm{W}(+2 / K)$ for $\alpha=5 \%$, but as yet no theoretical justification of it has been found. In section 5 it is proved that the reason is similar to the one given for case $K=1$. More recently, Schaarschmidt et al. ${ }^{20}$ indicate that, according to their results, method $\mathrm{W}(+1)$ is better than method $\mathrm{W}(+2 / K)$ for $K \geq 6$ and $\alpha=5 \%$.

Other methods exist which are operationally more complicated than the adjusted Wald method and which appear to produce better results. In some cases (Newcombe ${ }^{16}$ for $K=2$ and $\Sigma \beta_{i}=0$; Newcombe ${ }^{21}$ for $K=4$ and $\Sigma \beta_{i}=0$; Zou et $a l^{22}$ for any value of $\mathrm{K}$ and $\Sigma \beta_{i}$ ), the proportions $p_{i}$ are replaced by the extremes of Wilson's CI for the values $p_{i}$. In others (Beal ${ }^{23}$ for $K=2$; Tebbs and Roths ${ }^{14}$ for $K \geq 2$ ) the $K-1$ nuisance parameters are replaced by bayesian type estimators.

\section{Score method}

The aim of this section is to determine the value of the score statistic $z_{0}=(\bar{L}-\lambda) / \sqrt{V_{0}}$, where $V_{0}=\sum \beta_{i}^{2} \hat{p}_{i} \hat{q}_{i} / n_{i}, \hat{p}_{i}$ are the estimators of maximum likelihood (under $H_{0}$ ) of $p_{i}$ and

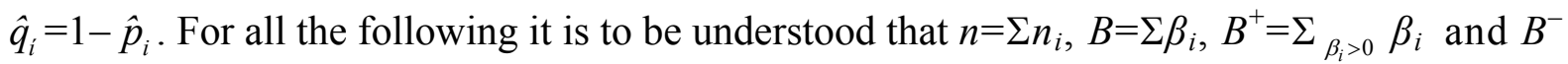
$=\Sigma_{\beta_{i}<0} \beta_{i}$. Observe that $B^{+}-B^{-}=\Sigma\left|\beta_{i}\right|, B^{+}+B^{-}=B$ and that $B^{-} \leq \lambda, \bar{L}, B \leq B^{+}$(since $0 \leq p_{i}$, $\left.\bar{p}_{i} \leq 1\right)$. Therefore, $-\Sigma\left|\beta_{i}\right| \leq \bar{L}-\lambda \leq+\Sigma\left|\beta_{i}\right|$. In Appendix A1 the following results, based on 
the key expression below, are proved:

$$
y=n+(B-2 \lambda) C-\Sigma R_{i}=0 \text { where }\left\{\begin{array}{l}
C=z^{2} /(\bar{L}-\lambda) \\
R_{i}^{2}=n_{i}^{2}+2 n_{i} \beta_{i} b_{i} C+\beta_{i}^{2} C^{2} . \\
b_{i}=1-2 \bar{p}_{i}
\end{array}\right.
$$

If $\bar{L}=\lambda$, it is obvious that $\hat{p}_{i}=\bar{p}_{i}$ and $z_{0}=0$. If $\bar{L} \neq \lambda$, then $z_{0}^{2}$ is the sole solution different from zero for the equation $y\left(z_{0}^{2}\right)=0$, and moreover:

$$
z_{0}=(\bar{L}-\lambda) / \sqrt{V_{0}} \text { where } V_{0}=\sum \beta_{i}^{2} \hat{p}_{i} \hat{q}_{i} / n_{i} \text { and } \hat{p}_{i}=\left(n_{i}+\beta_{i} C-R_{i}\right) / 2 \beta_{i} C \text {, }
$$

Searches for the value $z_{0}^{2}$ is made easier if it is borne in mind that:

$$
\frac{4(\bar{L}-\lambda)^{2}}{\sum \beta_{i}^{2} / n_{i}} \leq z_{0}^{2} \leq\left\{\begin{array}{ll}
T(\bar{L}-\lambda) /\left(\lambda-B^{-}\right) & \text {if } \bar{L}>\lambda \\
(n-T)(\bar{L}-\lambda) /\left(\lambda-B^{+}\right) & \text {if } \bar{L}<\lambda
\end{array},\right.
$$

where $T=\sum_{\beta_{i}>0} x_{i}+\sum_{\beta_{i}<0}\left(n_{i}-x_{i}\right)$. Alternatively, if the researcher does not wish to know the value of $z_{0}$, but only to know if the test has significance to the error $\alpha$, then $\mathrm{s} / \mathrm{he}$ need only apply the following rule based on expression (2):

$$
\text { Decide } H_{1} \Leftrightarrow y\left\{C=z_{\alpha / 2}^{2} /(\bar{L}-\lambda)\right\} \geq 0
$$

which simplifies the calculations enormously. For example, if one wishes to carry out the test of interaction $H_{0}: L_{l}=0$ vs. $H_{1}: L_{l} \neq 0$ in Table 1 to the error $\alpha=5 \%$, then $\lambda=B=0, z_{2.5 \%}^{2}=1.96^{2}$, $\bar{L}_{1}=-2 / 30, C=-15 \times 1.96^{2}, R_{i}^{2}=30\left\{30+1.96^{2}\left(a_{i}+7.5 \times 1.96^{2}\right)\right\}, a_{i}=10,2,-24$ and 8 for $i=1,2,3$ and 4 respectively and $y(C)=120-\Sigma R_{i}=-129.866<0$, for which reason the test is not significant. The intensity of the calculations is similar to that of Wald's test. In Appendix A2 it is proved that the statistic $z_{0}^{2}$ is equal to the classic chi-square statistic.

Another common aim is to obtain the score $\mathrm{CI}\left(\mathrm{CI}_{0}\right)$ for $L$. To this end it is sufficient to make $z_{0}^{2}=z_{\alpha / 2}^{2}$ in expression (2) and to determine both solutions $B^{-} \leq \lambda_{1}<\bar{L}<\lambda_{2} \leq B^{+}$of equation $y(\lambda)=0$ (see Appendix A3). If there is no solution $\lambda_{1}\left(\lambda_{2}\right)$ then $\lambda_{1}=B^{-}\left(\lambda_{2}=B^{+}\right)$. Table 3 indicate the values $z_{0}$ and/or the intervals $C I_{0}$ for the contrasts and/or effects in Tables 1 and 2 
(note that the contrasts L2 and L3 are significant and that there is no interaction between them). Similarly when the aim is to obtain the CI for $\beta_{K}$ in fixed values of $\lambda, \beta_{i} \neq \beta_{K}$ and $z_{0}^{2}=z_{\alpha / 2}^{2}$

Note that all the above contains the cases of one proportion $(K=1)$, of the difference between two proportions $\left(L=d=p_{2}-p_{1}\right)$ and of the risk ratio $\left(L=p_{2}-R p_{1}\right.$ and $\lambda=0)$ as special results. In particular, the tests and CI's of $\mathrm{Mee}^{24}$ for $d$ and of Koopman $^{25}$ for $R$ are special cases of the general case $L$. Similarly, the present proof that $z_{0}^{2}=\chi_{0}^{2}$ contains the proofs of $\mathrm{Nam}^{26}$ and Gart and $\mathrm{Nam}^{27}$ for $d$ and $R$ respectively as special cases. Expression (2) was proved by Martín and Herranz ${ }^{28}$ for case $d$.

\section{General and adjusted Wald -type approximations.}

In order to simplify the solution of equation (2) in $z_{0}^{2}$ (for the test) or in $\lambda$ (for the $\mathrm{CI}$ ), it is advisable to obtain approximate expressions of that equation. In Appendix A4 it is shown that, by expanding the term $R_{i}$ in Maclaurin series, expression (2) is converted to the following:

$$
(\bar{L}-\lambda)^{3}-z_{0}^{2}(\bar{L}-\lambda) V_{1}+z_{0}^{4} V_{2} \simeq 0, \text { where } V_{2}=\sum \frac{\beta_{i}^{3} \bar{p}_{i} \bar{q}_{i} b_{i}}{n_{i}^{2}}
$$

If one retains only the terms of order $\mathrm{O}\left(n_{i}\right) \geq-1$ and divides by $(\bar{L}-\lambda)$ one obtains Wald's classic solutions for expression (1). If one only retains the terms of $\mathrm{O}\left(n_{i}\right) \geq-2$, one replaces $z_{0}^{4} \simeq z_{0}^{2}(\bar{L}-\lambda)^{2} / V_{1}$ and divides by $(\bar{L}-\lambda)$, then $0 \simeq(\bar{L}-\lambda)^{2} V_{1}+(\bar{L}-\lambda) V_{2} z_{0}^{2}-$ $V_{1} z_{0}^{2}$ is obtained. From this one can deduce the following approximate statistic and CI:

$$
z_{2}=\frac{\bar{L}-\lambda}{\sqrt{V_{1}-(\bar{L}-\lambda) V_{2} / V_{1}}}, C I_{2}: \bar{L}+z_{\alpha / 2}^{2} \frac{V_{2}}{2 V_{1}} \pm z_{\alpha / 2} \sqrt{V_{1}+z_{\alpha / 2}^{2}\left(\frac{V_{2}}{2 V_{1}}\right)^{2}}
$$

In Table 3 the values of $z_{1}$ and $z_{2}$ for the contrasts in Table 1 are set out. It can be seen that both are near the real value $z_{0}$, and that $z_{2}$ is the best option. Something similar occurs 
with the intervals $C I_{1}$ and $C I_{2}$ for the effects $L$ of Tables 1 and 2 (see Table 3): $C I_{2}$ is the best option.

As has already been stated, the adjusted Wald heuristic methods $\mathrm{W}\left(+z_{\alpha / 2}^{2} / 2 K\right)$ have their origin in the proposal by Agresti and Coull ${ }^{15}$ for case $K=1$. These authors show that the center of Wilson's CI (which is the score CI for a proportion) is equal to the center of the adjusted Wald CI $\mathrm{W}\left(+z_{\alpha / 2}^{2} / 2\right)$, and this is the reason that this performs so well. On the basis of the approximations of this section it is now possible to prove that that is what occurs approximately in the case $K>1$. In Appendix A5 it is proved that the adjusted Wald method $\mathrm{W}\left(+c_{i}\right)$ where:

$$
c_{i}=\frac{n_{i} z_{\alpha / 2}^{2}}{2\left(K n_{i}-z_{\alpha / 2}^{2}\right)}=\frac{n_{i} h}{n_{i}-2 h} \simeq h \text { where } h=\frac{z_{\alpha / 2}^{2}}{2 K},
$$

has a center which is approximately the same as that of $C I_{2}$ in the expression (7). Note that by making $c_{i} \simeq h$, the adjusted Wald method $W(+h \simeq 2 / K)$ proposed by Price and Bonett ${ }^{18}$ for $\alpha=5 \%$ is obtained.

Table 3 sets out the CI obtained using the adjusted Wald methods $W\left(+c_{i}\right)$ and $W(+h)$. It can be seen that both methods produce very similar results, with a slight advantage in favour of the adjusted Wald method $W(+h)$. Note also that both procedures estimate the real range (that of $C I_{0}$ ) better than its center and that, in the case of large samples as in Table 2, all the procedures yield practically the same result.

The previous approximations are correct only when the observed data do not belong to the border of the sample space, that is, when $0<x_{i}<n_{i}(\forall i)$. Otherwise, at the end of Appendix A4 it is shown that the correct solutions for the intervals $C I_{1}$ and $C I_{2}$ and the adjusted Wald methods $W\left(+c_{i}\right)$ and $W(+h)$ are the intervals $C I_{1}^{\prime}$ and $C I_{2}^{\prime}$ and the following adjusted Wald methods $\mathrm{W}\left(+c_{i}^{\prime}\right)$ and $\mathrm{W}\left(+h_{i}^{\prime}\right)$ (in all cases one must make $j=1$ and use the sign - in order to obtain the lower extreme, and make $j=2$ and use the sign + to obtain the upper extreme): 


$$
\begin{gathered}
C I_{1}^{\prime}: \bar{L}+\frac{z_{\alpha / 2}^{2}}{2\left(N_{j}+z_{\alpha / 2}^{2}\right)}\left\{B_{j} \pm \sqrt{B_{j}^{2}+4\left(N_{j}+z_{\alpha / 2}^{2}\right) V_{1}}\right\} \\
C I_{2}^{\prime}: \bar{L}+\frac{z_{\alpha / 2}^{2}}{2\left(N_{j}+z_{\alpha / 2}^{2}\right)}\left\{B_{j}+\left(N_{j}+z_{\alpha / 2}^{2}\right) \frac{V_{2}}{V_{1}} \pm \sqrt{\left.\left\{B_{j}-\left(N_{j}+z_{\alpha / 2}^{2}\right) \frac{V_{2}}{V_{1}}\right\}^{2}+4\left(N_{j}+z_{\alpha / 2}^{2}\right) V_{1}\right\}}\right. \\
c_{i}^{\prime}=\frac{z_{\alpha / 2}^{2}}{2} \cdot \frac{n_{i}\left\{\frac{1}{K}+\frac{\delta_{i j} n_{i}}{N_{j}+z_{\alpha / 2}^{2}}\right\}}{n_{i}-z_{\alpha / 2}^{2}\left\{\frac{1}{K}+\frac{\delta_{i j} n_{i}}{N_{j}+z_{\alpha / 2}^{2}}\right\}} \text { and } h_{i}^{\prime}=\frac{z_{\alpha / 2}^{2}}{2} \cdot \frac{1+\delta_{i j} K}{K},
\end{gathered}
$$

where $N_{j}=\sum_{i} \delta_{i j} n_{i}, B_{j}=\sum_{i} \delta_{i j} b_{i} \beta_{i}, \delta_{i l}=1$ in all the subscripts $i$ verifying $\left\{\bar{p}_{i}=0\right.$ and $\left.\beta_{i}<0\right\}$ or $\left\{\bar{p}_{i}=1\right.$ and $\left.\beta_{i}>0\right\}\left(\delta_{i l}=0\right.$ otherwise $)$ and $\delta_{i 2}=1$ in all the subscripts $i$ verifying $\left\{\bar{p}_{i}=0\right.$ y $\left.\beta_{i}>0\right\}$ or $\left\{\bar{p}_{i}=1\right.$ and $\left.\beta_{i}<0\right\} \quad\left(\delta_{i 2}=0\right.$ otherwise $)$. Note that $C I_{1}^{\prime} \equiv C I_{1}, C I_{2}^{\prime} \equiv C I_{2}, \mathrm{~W}\left(+c_{i}^{\prime}\right) \equiv \mathrm{W}\left(+c_{i}\right)$ and $\mathrm{W}\left(+h_{i}^{\prime}\right) \equiv \mathrm{W}(+h)$ when $0<\bar{p}_{i}<1(\forall i)$ and that in $C I_{2}^{\prime}$ it is understood that $V_{2} / V_{l}=0$ when $V_{l}=0$.

\section{Coherence of the inferences: properties of convexity.}

In order for an $S$ statistic (such as $z_{0}$ ) to be useful in the inference it is necessary for it to verify certain coherence properties. This section aims to analyze these.

Barnard $^{29}$ recommended that the critical regions should be convex for the classic test $H_{0}: d=0$, and this means that the $S$ statistic should increase (decrease) in $\bar{p}_{2}\left(\bar{p}_{1}\right)$, although this increase or decrease need not be strict. Röhmel and Mansmann ${ }^{30}$ justified the fact that the same should occur in the more general case of $H_{0}: d=\delta$. In the present case $\left(H_{0}: L=\lambda\right)$ the statistic $S$ should increase (decrease) in the $\bar{p}_{i}$ values where $\beta_{i}>0\left(\beta_{i}<0\right)-$ i.e meaning $S$ should verify the property of spatial convexity- and this means that the CRs will present no gaps. In Annex A5 it is proved that $z_{0}$ verify this property. The proof contains two special cases: that of the difference $H_{0}: d=p_{2}-p_{1}=\delta$ (proved by Martín and Herranz ${ }^{31}$ ) and that of the risk ratio $H_{0}: p_{2}-R p_{1}=p_{2}-\rho p_{1}=0$ (for which there is no proof.).

Röhmel and Mansmann ${ }^{32}$ showed it was better for the p-value $P(\delta)$ for the test $H_{0}$ : $d=p_{2}-p_{1} \leq \delta$ to increase in $\delta$. In general, in order for test $H_{0}: L=\lambda$ based on $S$ to be coherent its 
p-value $P(\lambda)$ must increase (decrease) in $\lambda$ when $\lambda<\bar{L}(\lambda>\bar{L})$. This means that the $S$ statistic should decrease in $\lambda$, that is, that $S$ should verify the property of parametric convexity in $\lambda$. The verification of this property is what guarantees that inverting the test using the equality $S^{2}=z_{\alpha / 2}^{2}$ is equivalent to resolving the equality $S^{2} \leq z_{\alpha / 2}^{2}$ and yields a CI for $\lambda$ which presents no gaps. Similarly, in order for the CI for $\beta_{i}$ to be coherent it is necessary for $S$ to increase in $\beta_{i}$ (parametric convexity in $\beta_{i}$ ). In Annex A5 it is proved that $z_{0}$ verify both properties of parametric convexity (and it contains, as a special case, case $d$ of Martín and Herranz $\left.^{31}\right)$.

To summarize what has been said, any $S$ statistic should verify the following properties $\left(z_{0}\right.$ verify them):

$$
\frac{d S}{d \bar{p}_{i}}\left\{\begin{array}{l}
\geq 0 \text { si } \beta_{i}>0 \\
\leq 0 \text { si } \beta_{i}<0
\end{array}, \quad \frac{d S}{d \lambda} \leq 0, \quad \frac{d S}{d \beta_{i}} \geq 0\right.
$$

\section{Simulation study.}

In this section method $\mathrm{W}(+2 / K)$ (the best adjusted Wald method known at present) and the 8 new methods proposed in this paper $-\mathrm{S}$ (scores), $C I_{2}, C I_{1}^{\prime}, C I_{2}^{\prime}, \mathrm{W}\left(+c_{i}\right), \mathrm{W}(+h), \mathrm{W}\left(+c_{i}^{\prime}\right)$ and $\mathrm{W}\left(+h_{i}^{\prime}\right)-$ will be compared; the classic method of Wald $\mathrm{W}(+0) \equiv C I_{l}$ is excluded because, as has been said, it is known to behave badly.

For the $100(1-\alpha) \% \mathrm{CI}$, the actual probability of coverage $R$ and the expected interval width $W$ for fixed values of $p_{i}$ are defined by:

$$
R=\sum_{x_{1}=0}^{n_{1}} \sum_{x_{2}=0}^{n_{2}} \ldots \sum_{x_{K}=0}^{n_{K}} \prod_{i=1}^{K}\left(\begin{array}{c}
n_{i} \\
x_{i}
\end{array}\right) p_{i}^{x_{i}} q_{i}^{n_{i}-x_{i}} I\left(x_{1}, x_{2}, \ldots, x_{K}\right) \text { and } W=\sum_{x_{1}=0}^{n_{1}} \sum_{x_{2}=0}^{n_{2}} \ldots \sum_{x_{K}=0}^{n_{K}} \prod_{i=1}^{K}\left(\begin{array}{c}
n_{i} \\
x_{i}
\end{array}\right) p_{i}^{x_{i}} q_{i}^{n_{i}-x_{i}}\left(L_{S}-L_{I}\right)
$$

where $I\left(x_{1}, x_{2}, \ldots, x_{K}\right)=1$ if the $\mathrm{CI}\left(L_{I}, L_{S}\right)$ occasioned by the outcomes $\left(x_{1}, x_{2}, \ldots, x_{K}\right)$ contains $L=\Sigma \beta_{i} p_{i}$ and $I\left(x_{1}, x_{2}, \ldots, x_{K}\right)=0$ otherwise. For each set of values $\left(n_{i}, \beta_{i}\right)$ in Table 4, 10,000 sets of $p_{i}^{\prime} \mathrm{s}$ were randomly generated from the uniform $[0,1]$ distribution, and one of the previous methods was used to compute $W$ and $R$. The mean of $R$ (Rmean) and $W$ (Wmean), the minimum 
of $R$ (Rmin) and the percentage of $R$ that fell below 93\% (Rbelow93) in the 10,000 sets of $p_{i}{ }^{\prime} \mathrm{s}$ were computed for $1-\alpha=95 \%$. It is desirable for Rmean to be $95 \%$ on average (the method will be conservative if Rmean is greater than 95\%, and if not it will be liberal), for Rmin to be as close as possible to $95 \%$, for $W$ mean to be as small as possible and, finally, for the method to have few liberal "failures" (that is, for Rbelow93 to be as small as possible).

Table 4 shows the results for methods $\mathrm{W}(+2 / K), \mathrm{W}\left(+h_{i}^{\prime}\right)$ and $\mathrm{S}$ for a $\mathrm{CI}$ of $95 \%$. The results for the other methods are excluded (these may be requested from the authors) as we have determined that the methods $C I_{2}, C I_{1}^{\prime}$ y $C I_{2}^{\prime}$ fail a great deal, that the methods $\mathrm{W}\left(+c_{i}\right)$ and $\mathrm{W}(+h)$ function worse than $\mathrm{W}\left(+c_{i}^{\prime}\right)$ and $\mathrm{W}\left(+h_{i}^{\prime}\right)$ and, finally, that method $\mathrm{W}\left(+c_{i}^{\prime}\right)$ is too conservative. From these first results we can extract two commentaries of interest. In the first place, it is surprising that, given that method $\mathrm{W}\left(+h_{i}^{\prime}\right)$ is an approximation of method $\mathrm{W}\left(+c_{i}^{\prime}\right)$, and the latter is in turn an approximation of method $\mathrm{CI}_{2}^{\prime}$, the previous results indicate that the first is better than the second which in turn is better than the third. Secondly, and given that $\mathrm{W}\left(+c_{i}\right)$ and $\mathrm{W}(+h)$ are worse methods than $\mathrm{W}\left(+c_{i}^{\prime}\right)$ and $\mathrm{W}\left(+h_{i}^{\prime}\right)$ respectively, it is necessary to point out the importance of defining CI differently when the outcomes are extreme $\left(x_{i}=0\right.$ or $\left.x_{i}=n_{i}\right)$ to when they are not $\left(0<x_{i}<n_{i}\right)$.

From Table 4 we can deduce that the best option is S (except when all the sample sizes are equal to 10) because compared to the other two methods it is more balanced, it has an equal or smaller Wmean, its liberal failures are almost always lower (that is, its Rbelow93 is almost always smaller) and its value for $\operatorname{Rmin}$ is almost always closer to $95 \%$. It can also be seen that method $\mathrm{W}(+2 / K)$ is slightly conservative and that $\mathrm{W}\left(+h_{i}^{\prime}\right)$ is always very conservative (especially in $K=4$ ), and as a result the second of the two usually has few failures (especially in $K=4$ ) and an excessively large value for Wmean. Finally, we should point out that although the method $\mathrm{W}(+2 / K)$ contains some desirable features, it is not reliable because its value for Rbelow93 can be very large; in contrast, method $\mathrm{W}\left(+h_{i}^{\prime}\right)$ is reliable, but is also very 
conservative. In general, these conclusions remain valid for $90 \%$ and $99 \%$ CI's (the data may be requested from the authors), although $\mathrm{W}(+2 / \mathrm{K})$ now behaves very well for $1-\alpha=90 \%$ and very badly for $1-\alpha=99 \%$.

\section{Conclusions.}

Asymptotic inferences (tests or confidence intervals) relative to independent binomial proportions are very frequent in applied research, but until now the research has centered almost entirely on cases with one simple proportion $p$ and on the difference $\left(d=p_{2}-p_{1}\right)$ or the ratio $\left(R=p_{2} / p_{1}\right)$ between two proportions. Surprisingly, the case of a linear of $K$ proportions $\left(L=\Sigma \beta_{i} p_{i}\right)$ has received very little attention, despite its great practical importance ${ }^{14}$. Even more surprising is the fact that the problem has been approached till now only from the point of view of the confidence intervals obtained by the classic Wald method.

In this article the problem is looked at from the point of view of the score method (equivalent to the classic chi-square method), and proves the suitability of this method compared to the other 8 procedures. Because the application of the method requires an iterative procedure, the reader may apply the free program obtainable at http://www.ugr.es/local/bioest/Z_LINEAR_K.EXE.

The paper also provides a theoretical proof of the heuristic result that Wald's $95 \%$ confidence interval improves if $2 / K$ successes and failures are added to each sample. In addition, at the end of section 5 the rule is generalized, so that a simple and reliable (although conservative) CI consists in applying Wald's classic CI from expression (1) and adding $h_{i}^{\prime}$ successes and failures to each sample, a quantity which is reduced to $z_{\alpha / 2}^{2} / 2 K$ when $0<x_{i}<n_{i}$ in all the samples (which is usually the case). When the values of $n_{i}$ and/or $K$ are small it may be more suitable to add $c_{i}=n_{i} z_{\alpha / 2}^{2} /\left[2\left(K n_{i}-z_{\alpha / 2}^{2}\right)\right]$ successes and failures.

The article also points out how important it is for any test statistic (such as $z_{0}$ ) to verify both spatial and parametric convexity. The first, so that the test behaves coherently. The 
second, so that the CI can be obtained by inverting the test by means of the equality $z_{0}^{2}=z_{\alpha / 2}^{2}$.

\section{Acknowledgments.}

This research was supported by the Ministerio de Educación y Ciencia, Spain, grant number MTM-2008-01697/MTM (with cofinancing by the FEDER) and by the Consejería de Innovación, Ciencia y Empresa, Junta de Andalucía, Spain, grant number FQM-01459.

\section{APPENDIX A}

\section{A1. Estimators of maximum likelihood and the score test.}

Since, under $H_{0}, \quad \operatorname{Pr} \quad\left(x_{1}, \quad \ldots, \quad x_{K} \mid \lambda=\Sigma \beta_{i} p_{i}\right)=\quad \prod\left(\begin{array}{c}n_{i} \\ x_{i}\end{array}\right) p_{i}^{x_{i}} q_{i}^{n_{i}-x_{i}}, \quad$ where $p_{K}=\left(\lambda-\sum_{i \neq K} \beta_{i} p_{i}\right) / \beta_{K}$, then $\ell=\ln \operatorname{Pr}\left(x_{1}, \ldots, x_{K} \mid \lambda=\Sigma \beta_{i} p_{i}\right) \propto \Sigma x_{i} \ln p_{i}+\Sigma\left(n_{i}-x_{i}\right) \ln \left(1-p_{i}\right)$.

When $\bar{L}=\lambda$, the restricted estimators of maximum likelihood $\hat{p}_{i}$ are equal to the classic unrestricted $\bar{p}_{i}$ ones.

When $\hat{L} \neq \lambda$, because $d p_{K} / d p_{i}=\beta_{i} / \beta_{K}$ then the $\hat{p}_{i}$ are the solutions to $d \ell / d p_{i}=$ $\left(\partial \ell / \partial p_{i}\right)-\left(\beta_{i} / \beta_{K}\right) /\left(\partial \ell / \partial p_{K}\right)=n_{i}\left(\bar{p}_{i}-p_{i}\right) / p_{i} q_{i}-\left(\beta_{i} / \beta_{K}\right) n_{K}\left(\bar{p}_{K}-p_{K}\right) / p_{K} q_{K}=0(\forall i)$, that is:

$$
\frac{n_{i} \bar{p}_{i}}{\beta_{i} p_{i}}+\frac{n_{i} \bar{q}_{i}}{\beta_{i} q_{i}}=\frac{n_{i}\left(\bar{p}_{i}-p_{i}\right)}{\beta_{i} p_{i} q_{i}}=C(\forall i)
$$

where $C$ is a constant to be determined. From the above it can be deduced that $\hat{p}_{i}=\left(n_{i}+\beta_{i} C \pm R_{i}\right) / 2 \beta_{i} C$, where $R_{i}^{2}=n_{i}^{2}+2 n_{i} \beta_{i} b_{i} C+\beta_{i}^{2} C^{2}$ and $b_{i}=1-2 \bar{p}_{i}$.

In order to see which of the two solutions $\hat{p}_{i}(+)$ or $\hat{p}_{i}(-)$ is the appropriate one, one must remember that $R_{i}^{2} \in\left(n_{i} \pm \beta_{i} C\right)^{2}$, because $0 \leq \bar{p}_{i} \leq 1$, so that $R_{i} \geq\left|n_{i}-\right| \beta_{i} C|| \geq-n_{i}+\left|\beta_{i} C\right|$. When $\beta_{i} C>0$, this implies that $\hat{p}_{i}(+) \geq 1$, which is impossible unless $\hat{p}_{i}(+)=+1$. But if this is so, it is because $\bar{p}_{i}=+1, n_{i}=\beta_{i} C$-from expression (A1)- and $R_{i}=0$; hence $\hat{p}_{i}(+)=\hat{p}_{i}(-)=+1$. Similarly, if $\beta_{i} C<0$ then $\hat{p}_{i}(+) \leq 0$ which is also impossible unless $\hat{p}_{i}(+)=\hat{p}_{i}(-)=0$. Hence 
the solution will always be $\hat{p}_{i}(-)$. This means that $2 \beta_{i} \hat{p}_{i} C=n_{i}+\beta_{i} C-R_{i}$, so that by adding in $i$, and bearing in mind that $\hat{L}=\sum \beta_{i} \hat{p}_{i}=\lambda$, that makes $C$ the solution to the equation:

$$
y(C)=n+(B-2 \lambda) C-\Sigma R_{i}=0 .
$$

The constant $C$ may be expressed in the following ways:

$$
C=\frac{\bar{L}-\lambda}{V_{0}}=\frac{z_{0}^{2}}{\bar{L}-\lambda}=\frac{1}{K} \sum \frac{n_{i}\left(\bar{p}_{i}-\hat{p}_{i}\right)}{\beta_{i} \hat{p}_{i} \hat{q}_{i}}=\frac{\sum n_{i}\left(\bar{p}_{i}-\hat{p}_{i}\right)}{\sum \beta_{i} \hat{p}_{i} \hat{q}_{i}}=\frac{1}{B} \sum \frac{n_{i}\left(\bar{p}_{i}-\hat{p}_{i}\right)}{\hat{p}_{i} \hat{q}_{i}} .
$$

In order to obtain the first equality one need only note that, from expression (A1), $\beta_{i}\left(\bar{p}_{i}-\hat{p}_{i}\right)=\beta_{i}^{2} \hat{p}_{i} \hat{q}_{i} C / n_{i}$, so that by adding in $i:(\bar{L}-\lambda)=C \sum \beta_{i}^{2} \hat{p}_{i} \hat{q}_{i} / n_{i}=C V_{0}$. The other equalities are obtained in a similar way, except the second, which proceeds from the fact that $z_{0}^{2}=(\bar{L}-\lambda)^{2} / V_{0}$. From expressions (A1) and (A3) it can be deduced that

$$
\operatorname{Sign}(C)=\operatorname{Sign}\left(\bar{p}_{i}-\hat{p}_{i}\right) / \beta_{i}=\operatorname{Sign}(\bar{L}-\lambda),
$$

and that in the contrasts $(B=0), \sum n_{i}\left(\bar{p}_{i}-\hat{p}_{i}\right) / \hat{p}_{i} \hat{q}_{i}=0$. Also note that $z_{0}^{2}=n_{i}\left(\bar{p}_{i}-\hat{p}_{i}\right)(\bar{L}-\lambda)$ $/ \beta_{i} \hat{p}_{i} \hat{q}_{i}(\forall i)$

In order to prove that, when $\bar{L} \neq \lambda$, the equation (A2) has a unique solution $C \neq 0$, it is necessary to study function $y(C)$. Note that $y(C=0)=0$, so that $C=0$ is always a false solution to equation (A3). On the other hand, $d y / d C=(B-2 \lambda)-\Sigma \beta_{i}\left(\beta_{i} C+n_{i} b_{i}\right)=0$ will provide the extremes $\bar{C}$ of function $y(C)$. If they exist, they give rise to a maximum, given that $d y^{2} / d C^{2}=$ $-4 \sum \beta_{i}^{2} n_{i} \bar{p}_{i} \bar{q}_{i} / R_{i}^{3}<0$. However, since:

$$
\lim _{C \rightarrow \pm \infty} \frac{y(C)}{C}=(B-2 \lambda) \mp \sum\left|\beta_{i}\right|=\left\{\begin{array}{l}
2\left(B^{-}-\lambda\right)=m^{+} \text {if } C \rightarrow+\infty \\
2\left(B^{+}-\lambda\right)=m^{-} \text {if } C \rightarrow-\infty
\end{array},\right.
$$

then $y(C)$ has two oblique asymptotes from slope $m^{+}$and $m^{-}$and from equations $y=m^{ \pm} C+$ $h^{ \pm}$, where:

$$
h^{ \pm}=\lim _{C \rightarrow \pm \infty}\left\{y(C)-m^{ \pm} C\right\}=\lim _{C \rightarrow \pm \infty} \sum\left(n_{i} \pm\left|\beta_{i}\right| C-R_{i}\right)=\lim _{C \rightarrow \pm \infty} \frac{\left( \pm 2 n_{i}\left|\beta_{i}\right|-2 n_{i} \beta_{i} b_{i}\right) C}{n_{i} \pm\left|\beta_{i}\right| C+R_{i}}=
$$




$$
=\sum n_{i}\left(1 \mp s_{i} b_{i}\right)=\left\{\begin{array}{ll}
2 T=h^{+} & \text {if } C \rightarrow+\infty \\
2(n-T)=h^{-} & \text {if } C \rightarrow-\infty
\end{array},\right.
$$

where $s_{i}=\operatorname{Sign}\left(\beta_{i}\right)$ and $T$ is the value referred to in expression (4). But, if $A_{i}=\beta_{i} C+n_{i} b_{i}$ :

$$
y(C)-m^{ \pm} C-h^{ \pm}=\sum\left\{ \pm s_{i} A_{i}-R_{i}\right\}=-\sum \frac{4 n_{i}^{2} \bar{p}_{i} \bar{q}_{i}}{ \pm s_{i} A_{i}+R_{i}}<0,
$$

because as $R_{i}^{2}=A_{i}^{2}+4 n_{i}^{2} \bar{p}_{i} \bar{q}_{i}$ then $R_{i} \geq\left|A_{i}\right| \geq \pm A_{i}= \pm s_{i} A_{i}$ and the denominator of the previous fraction is positive. Expression (A5) indicates that function $y(C)$ is always found below the two asymptotes and, from what was stated above, it will have a maximum in $C=\bar{C}$. Because, moreover, it cuts the horizontal axis at $C=0$, it can be deduced that it also ought to cut that axis at another point $C=C_{0} \neq 0$ which will be $C_{0}>0\left(C_{0}<0\right)$ when $\bar{L}>\lambda(\bar{L}<\lambda)$. In addition the solution $C=C_{0}$ will have to be sought where the asymptotes cut the horizontal axis: $-h^{+} / m^{+}=$ $T /\left(\lambda-B^{-}\right)$and $-h^{-} / m^{-}=-(n-T) /\left(B^{+}-\lambda\right)$. Finally, since $\hat{p}_{i} \hat{q}_{i} \leq 1 / 4$ then, from the first equality of (A3), $|C| \geq 4|\bar{L}-\lambda| /\left(\sum \beta_{i}^{2} / n_{i}\right)$. As a result it can be affirmed that the equation (A2) has only one solution $C_{0} \neq 0$ which is contained between the following bounds:

$$
\left\{\begin{array}{l}
\text { If } \bar{L}>\lambda: 4(\bar{L}-\lambda) /\left(\sum \beta_{i}^{2} / n_{i}\right) \leq C_{0} \leq T /\left(\lambda-B^{-}\right) \\
\text {If } \bar{L}<\lambda:-(n-T) /\left(B^{+}-\lambda\right) \leq C_{0} \leq 4(\bar{L}-\lambda) /\left(\sum \beta_{i}^{2} / n_{i}\right)
\end{array} .\right.
$$

Once the value $C_{0}$ has been determined then $z_{0}^{2}=C_{0}(\bar{L}-\lambda)$.

In order to obtain the value $z_{0}^{2}$ directly, without having to obtain the value of $C_{0}$ previously, one need only replace $C$ with $z_{0}^{2} /(\bar{L}-\lambda)$; hence the expressions (2) and (4). By making this change in (A2) and multiplying the whole expression by $(\bar{L}-\lambda)$, the following, more general, equation is obtained:

$$
f=n(\bar{L}-\lambda)+(B-2 \lambda) z_{0}^{2}-\operatorname{Sign}(\bar{L}-\lambda) \sum \bar{R}_{i}=0
$$

where $\bar{R}_{i}^{2}=n_{i}^{2}(\bar{L}-\lambda)^{2}+\beta_{i}^{2} z_{0}^{4}+2 n_{i} \beta_{i} b_{i} z_{0}^{2}(\bar{L}-\lambda)$. With this format $\hat{p}_{i}=\left\{n_{i}(\bar{L}-\lambda)+\beta_{i} z_{0}^{2}-\right.$ 
Sign $\left.(\bar{L}-\lambda) \bar{R}_{i}\right\} / 2 \beta_{i} z_{0}^{2}$. By solving $f=0$ the value of $z_{0}^{2}$ is obtained.

Alternatively, if the researcher does not wish to obtain the p-value of the test, but only carry out the test to error $\alpha$, the calculations become very much simpler, because it is not necessary to resolve equation (2). In effect, as $H_{i}$ will be decided when $z_{0}^{2}=C_{0}(\bar{L}-\lambda) \geq z_{\alpha / 2}^{2}$, then the test will be significant if $C_{0} \geq z_{\alpha / 2}^{2} /(\bar{L}-\lambda)$ or $C_{0} \leq z_{\alpha / 2}^{2} /(\bar{L}-\lambda)$ when $\bar{L}>\lambda$ or $\bar{L}<\lambda$ respectively, that is, when $y\left\{C=z_{\alpha / 2}^{2} /(\bar{L}-\lambda)\right\} \geq 0$ in expression (2); this is due to the fact that $y(C) \geq 0$ for $C$ between 0 and $C_{0}$, as has been indicated above. If the format for expression (A6) is adopted, the following alternative statement is obtained: decide $H_{1} \Leftrightarrow f\left(z_{\alpha / 2}^{2}\right) \geq 0$ (if $\bar{L}>\lambda$ ) or $f\left(z_{\alpha / 2}^{2}\right) \leq 0$ (if $\bar{L}<\lambda$ ).

\section{A2. The chi-square test.}

The traditional chi-square test is:

$$
\begin{gathered}
\chi_{0}^{2}=\sum\left\{\frac{\left(x_{i}-n_{i} \hat{p}_{i}\right)^{2}}{n_{i} \hat{p}_{i}}+\frac{\left(n_{i}-x_{i}-n_{i} \hat{q}_{i}\right)^{2}}{n_{i} \hat{q}_{i}}\right\}=\sum \frac{n_{i}\left(\bar{p}_{i}-\hat{p}_{i}\right)^{2}}{\hat{p}_{i} \hat{q}_{i}}=\sum \frac{n_{i}\left(\bar{p}_{i}-\hat{p}_{i}\right)}{\hat{p}_{i} \hat{q}_{i}} \beta_{i}\left(\bar{p}_{i}-\hat{p}_{i}\right) \\
=C \sum \beta_{i}\left(\bar{p}_{i}-\hat{p}_{i}\right)=C(\bar{L}-\lambda)=z_{0}^{2},
\end{gathered}
$$

where the last three equalities are due to expression (A1), to the fact that $\bar{L}=\sum \beta_{i} \bar{p}_{i}$ and $\lambda=\sum n_{i} \hat{p}_{i}$, and to expression (A3) respectively.

\section{A3. The score $\mathbf{C I}$.}

Because $z_{0}^{2}=C(\bar{L}-\lambda)$ then $\lambda=\bar{L}-z_{0}^{2} / C$. After substitution in expression (2), $y(C)=n+2 z_{0}^{2}+(B-2 \bar{L}) C-\sum R_{i}=0$ is obtained. In order to obtain a CI for $\lambda$ one only need make $z_{0}^{2}=z_{\alpha / 2}^{2}$, determine the two values $C=C_{1}>0$ and $C=C_{2}<0$ which satisfy the previous equation and calculate $\lambda_{i}=\bar{L}-z_{\alpha / 2}^{2} / C_{i}$. From this, $\lambda_{1} \leq \lambda \leq \lambda_{2}$ is the required solution. It is more direct to resolve equation (2) in $\lambda$ within the licit margins: $B^{-} \leq \lambda_{1}<\bar{L}<\lambda_{2} \leq B^{+}$. Alternatively equation (A7) can be used and resolved in $\lambda$. Based on expression (4), it can be seen that 
some more specific bounds where solutions $\lambda_{i}$ can be sought are $\bar{L}-z_{\alpha / 2} \sqrt{\sum \beta_{i}^{2} / 4 n_{i}} \leq \lambda_{I} \leq$ $\left(z_{\alpha / 2}^{2} B^{-}+T \bar{L}\right) /\left(z_{\alpha / 2}^{2}+T\right) \quad$ and $\quad\left\{z_{\alpha / 2}^{2} B^{+}+(n-T) \bar{L}\right\} /\left\{z_{\alpha / 2}^{2}+(n-T)\right\} \leq \lambda_{2} \leq \bar{L}+$ $z_{\alpha / 2} \sqrt{\sum \beta_{i}^{2} / 4 n_{i}}$

\section{A4. Approximations.}

Expanding $R_{i}$ in Maclaurin series for $C=0$ indicates that:

$$
R_{i} \simeq n_{i}+\beta_{i} b_{i} C+\frac{2 \beta_{i}^{2} \bar{p}_{i} \bar{q}_{i}}{n_{i}} C^{2}-\frac{2 \beta_{i}^{3} \bar{p}_{i} \bar{q}_{i} b_{i}}{n_{i}^{2}} C^{3}
$$

so that by substitution in expression (2) and by dividing by $2 C$ one obtains $0 \simeq(\bar{L}-\lambda)$ $-C V_{1}+C^{2} V_{2}$ with the $V_{i}$ as in expressions (1) and (6). By substituting $C=z_{0}^{2} /(\bar{L}-\lambda)$ and operating, expression (6) is obtained.

In section 5 it is shown that a CI with order $\mathrm{O}\left(n_{i}\right) \geq-2$ is given by expression (7). The present aim is to express its center $\bar{L}+\left(z_{\alpha / 2}^{2} / 2\right) V_{2} / V_{1}$ in Wald's traditional format, that is, to make it equal to $\bar{L}^{\prime}$ based on the increased observations $x_{i}^{\prime}=x_{i}+c_{i}$ and $n_{i}^{\prime}=n_{i}+2 c_{i}$, where $c_{i}$ are values to be determined. In order to do this approximately one must bear in mind that:

$$
\frac{V_{2}}{V_{1}}=\left\{\sum \frac{\beta_{i}^{2} \bar{p}_{i} \bar{q}_{i}}{n_{i}} \cdot \frac{\beta_{i} b_{i}}{n_{i}}\right\} / \sum \frac{\beta_{i}^{2} \bar{p}_{i} \bar{q}_{i}}{n_{i}} \simeq \frac{1}{K} \sum \frac{\beta_{i} b_{i}}{n_{i}},
$$

because $V_{2} / V_{1}$ is the weighted average of $\beta_{i} b_{i} / n_{i}$ and it will be approximately equal to its arithmetic average. Thus the center of the CI (7) will be:

$$
\bar{L}+\frac{z_{\alpha / 2}^{2}}{2} \cdot \frac{V_{2}}{V_{1}} \simeq \sum \beta_{i} \frac{x_{i}+h b_{i}}{n_{i}} \text { where } h=\frac{z_{\alpha / 2}^{2}}{2 K} .
$$

As the center of the adjusted Wald CI $W\left(+c_{i}\right)$ is $\Sigma \beta_{i}\left(x_{i}+c_{i}\right) /\left(n_{i}+2 c_{i}\right)$, then by making both expressions equal it is found that $c_{i}$ must verify the equality $\left(x_{i}+c_{i}\right) /\left(n_{i}+2 c_{i}\right)=\left(x_{i}+h b_{i}\right) / n_{i}$, and so $c_{i}=n_{i} h /\left(n_{l}-2 h\right)$ as indicated in section 5 . 
All the above is valid when $0<\bar{p}_{i}<1$, because when $\bar{p}_{i}=0$ or $1-$ that is, when $b_{i}= \pm 1-$ then $R_{i}=\left|n_{i}+b_{i} \beta_{i} C\right|$ and the serial development of expression (A8) yields a value of $n_{i}+b_{i} \beta_{i} C$ which is does not necessarily coincide with the previous one. For example, when $\bar{p}_{i}=0$ and $\beta_{i} C<0$ expression (A1) indicates that $\beta_{i} C=-n_{i} / \hat{q}_{i}$ and thus $0=n_{i}+\hat{q}_{i} \beta_{i} C \geq n_{i}+\beta_{i} C$ (because $\left.\hat{q}_{i} \leq 1\right)=n_{i}+b_{i} \beta_{i} C$ (because $\left.b_{i}=1\right)$; as a result $R_{i}=-\left(n_{i}+b_{i} \beta_{i} C\right)$ and not $n_{i}+b_{i} \beta_{i} C$ as expression (A8) indicated. The same result is obtained when $\bar{p}_{i}=1$ and $\beta_{i} C>0$. The conclusion is that $R_{i}=-\left(n_{i}+b_{i} \beta_{i} C\right)$ when $b_{i}= \pm 1$ and $b_{i} \beta_{i} C<0$, and otherwise the approximation of the expression (A8) may be applied. If with this new definition one proceeds as at the beginning of this annex, the following expression, which is more exact than (6), is obtained (in which one must make $j=1$ if $\bar{L}>\lambda$ and $j=2$ if $\bar{L}<\lambda)$ :

$$
0 \simeq N_{j}(\bar{L}-\lambda)^{3}+(\bar{L}-\lambda)^{2}\left\{(\bar{L}-\lambda)+B_{j}\right\} z_{0}^{2}-(\bar{L}-\lambda) V_{1} z_{0}^{4}+V_{2} z_{0}^{6}
$$

When $0<\bar{p}_{i}<1 \quad(\forall i)$, then $\delta_{i j}=0(\forall i, j), N_{j}=B_{j}=0$ and expression (A10) turns into expression (6). When $b_{i}= \pm 1$, that is, when the observed point falls in one of the corners of the sample space, then the following result (which can be shown to be the same as that of the score method) is obtained:

$$
z_{0}^{2}=\left\{\begin{array}{l}
\frac{(\bar{L}-\lambda) N_{1}}{\lambda-B^{-}} \text {if } \bar{L}>\lambda \\
\frac{(\bar{L}-\lambda) N_{2}}{\lambda-B^{+}} \text {if } \bar{L}<\lambda
\end{array} \quad \text { and } \quad \frac{N_{1} \bar{L}+z_{\alpha / 2}^{2} B^{-}}{N_{1}+z_{\alpha / 2}^{2}} \leq L \leq \frac{N_{2} \bar{L}+z_{\alpha / 2}^{2} B^{+}}{N_{2}+z_{\alpha / 2}^{2}}\right.
$$

In general, if one proceeds for expression (A10) as one did for expression (6) in section 5, expressions (9) and (10) are obtained. Lastly, if the center of the interval $\mathrm{CI}_{2}^{\prime}$ are equal to that of interval $C I_{1}$ with its data increased in $c_{i}^{\prime}$ (just as above with the center of the interval $C I_{2}$ ) expression (11) is obtained. 


\section{A5. Properties of convexity}

Let $S=z_{0}$ in expression (12) and let $\psi=\bar{p}_{i}, \lambda$ or $\beta_{i}$. Because $d z_{0}^{2} / d \psi=2 z_{0}\left(d z_{0} / d \psi\right)$, then the sign of $d z_{0}^{2} / d \psi$ is the same as (different to) the sign of $d z_{0} / d \psi$ when $\bar{L}>\lambda(\bar{L}<\lambda)$ because then $z_{0}>0\left(z_{0}<0\right)$. This means that the convexity properties (12) are verified for $z_{0}$ if $z_{0}^{2}$ verifies the expressions (12) when $\bar{L}>\lambda$, or the opposite ones when $\bar{L}<\lambda$. The aim is thus to calculate $d z_{0}^{2} / d \psi$

For expression (2), $\partial y / \partial \lambda=-2 C, \partial y / \partial \bar{p}_{i}=2 n_{i} \beta_{i} C / R_{i}, \partial y / \partial \beta_{i}=C\left\{R_{i}-A_{i}\right\} / R_{i}$ and:

$$
\frac{\partial y}{\partial C}=(B-2 \lambda)-\sum \frac{\beta_{i} A_{i}}{R_{i}}=D \text { where }-2 n \leq D C \leq 0,
$$

where the last statement is owed to the fact that $D C=(B-2 \lambda)-\Sigma \beta_{i} C A_{i} / R_{i}$ or, using expression (2), $D C=-n+\sum R_{i}-\sum \beta_{i} C A_{i} / R_{i}=-n+\sum n_{i} A_{i}^{\prime} / R_{i}$ where $A_{i}^{\prime}=n_{i}+b_{i} \beta_{i} C$; but as $R_{i}^{2}=A_{i}^{\prime 2}+$ $4 \beta_{i}^{2} C^{2} \bar{p}_{i} \bar{q}_{i}$ then $R_{i} \geq\left|A_{i}^{\prime}\right|,-1 \leq A_{i}^{\prime} / R_{i} \leq+1$ and $-2 n \leq D C \leq 0$. From which it can be deduced that:

$$
\operatorname{Sign}(D) \neq \operatorname{Sign}(C)=\operatorname{Sign}(\bar{L}-\lambda) \text {, }
$$

and the last statement is owed to what has been said in expression (A4).

Because $y=0, d y / d \psi=0=(\partial y / \partial \psi)+(\partial y / \partial C)(d C / d \psi)$, so that $d C / d \psi=-(\partial y / \partial \psi) /$ $(\partial y / \partial C)$ and so:

$$
\frac{d C}{d \lambda}=\frac{2 C}{D}, \frac{d C}{d \beta_{i}}=-\frac{C\left(R_{i}-A_{i}\right)}{D R_{i}}, \frac{d C}{d \bar{p}_{i}}=-\frac{2 n_{i} \beta_{i} C}{D R_{i}}
$$

Finally, as $z_{0}^{2}=C(\bar{L}-\lambda)$ then $d z_{0}^{2} / d \psi=\left(\partial z_{0}^{2} / \partial \psi\right)+(\bar{L}-\lambda)(d C / d \psi)$, so that by substituting expressions (A12):

$$
\frac{d z_{0}^{2}}{d \lambda}=C\left\{\frac{2(\bar{L}-\lambda)}{D}-1\right\}, \frac{d z_{0}^{2}}{d \bar{p}_{i}}=\beta_{i} C\left\{1-\frac{2 n_{i}(\bar{L}-\lambda)}{D R_{i}}\right\}, \frac{d z_{0}^{2}}{d \beta_{i}}=C\left\{\bar{p}_{i}-\frac{\left(R_{i}-A_{i}\right)(\bar{L}-\lambda)}{D R_{i}}\right\},
$$


where $R_{i} \geq A_{i}$ as indicated in section (A1). If, in expressions (A13) one bears in mind expressions (A11) it can be deduced that $z_{0}^{2}$ verifies expressions (12) when $\bar{L}>\lambda$ and the opposite ones when $\bar{L}<\lambda$.

\section{REFERENCES}

1. Brumback B, and Berg A. On effect-measure modification: relationships among changes in the relative risk, odds ratio, and risk difference. Statistics in Medicine 2008; 27: 3453-3465. DOI: $10.1002 / \operatorname{sim} .3246$.

2. Crans GG, Shuster JJ. How conservative is Fisher's exact test? A quantitative evaluation of the two-sample comparative binomial trial. Statistics in Medicine 2008; 27: 3598-3611. DOI: $10.1002 / \operatorname{sim} .3221$.

3. Herranz Tejedor I, Martín Andrés A. A numerical comparison of several unconditional exact tests in problems of equivalence based on the difference of proportions. Journal of Statistical Computation and Simulation 2008; 78: 969-981. DOI: 10.1002/sim.3373.

4. Kabaila P. Statistical properties of exact confidence intervals from discrete data using studentized test statistics. Statistics \& Probability Letters 2008; 78: 720-727. DOI: 10.1016/j.spl.2007.09.035.

5. Lang JB. Score and profile likelihood confidence intervals for contingency table parameters. Statistics in Medicine 2008; 27: 5975-5990. DOI: 10.1002/sim.3391.

6. Martín Andrés A. Comments on "Chi-squared and Fisher-Irwin tests of two-by-two tables with small sample recommendations". Statistics in Medicine 2008; 27: 1791-1795 (Reply in 1796-1796). DOI: 10.1002/sim.3169.

7. Martín Andrés A, Álvarez Hernández M. Comments on 'Active-control trials with binary data: a comparison of methods for testing superiority or non-inferiority using the odds ratio. Statistics in Medicine 2008; 27: 5799-5800. DOI: 10.1080/ 10629360601026386.

8. Martín Andrés A, Tapia Garcia JM, del Moral Ávila MJ. Two-tailed unconditional 
inferences on the difference of two proportions in cross-sectional studies. Communications in Statistic - Simulation and Computation 2008; 37: 455-465. DOI: 10.1080/ 03610910701812360.

9. Reiczigel J, Abonyi-Tóth Z, Singer J. An exact confidence set for two binomial proportions and exact unconditional confidence intervals for the difference and ratio of proportions. Computational Statistics and Data Analysis 2008; 52: 5046-5053. doi:10.1016/j.csda. 2008.04.032

10. Roussou V, Seifert B. A mixed approach for proving non-inferiority in clinical trials with binary endpoint. Biometrical Journal 2008; 50: 190-204. DOI: 10.1002/bimj.200710410.

11. Siqueira AL, Whitehead A, Todd S. Active-control trials with binary data: a comparison of methods for testing superiority or non-inferiority using the odds ratio. Statistics in Medicine 2008; 27: 353-370. DOI: 10.1002/sim.2975.

12. Vos PW, Hudson S. Problems with binomial two-sided tests and the associated confidence intervals. Australian \& New Zealand Journal of Statistics2008; 50: 81-89. DOI: 10.1111/ j.1467-842X.2007.00501.x.

13. Zou , Donner A. (2008). Construction of confidence limits about effect measures: A general approach. Statistics in Medicine 27: 1693-1702. DOI: 10.1002/sim.3095.

14. Tebbs JM, Roths SA. New large-sample confidence intervals for a linear combination of binomial proportions. Journal of Statistical Planning and Inference 2008; 138: 18841893. DOI: 10.1016/j.jspi.2007.07.008.

15. Agresti A, Coull BA. Approximate Is Better than "Exact" for Interval Estimation of Binomial Proportions. The American Statistician 1998; 52: 119-126.

16. Newcombe RG. Interval estimation for the difference between independent proportions: comparison of eleven methods. Statistics in Medicine 1998; 17: 873-890. DOI: 10.1002/(SICI)1097-0258(19980430). 
17. Agresti A, Caffo B. Simple and effective confidence intervals for proportions and difference of proportions result from adding two successes and two failures. The American Statistician 2000; 54: 280-288.

18. Price RM, Bonett DG. An improved confidence interval for a linear function of binomial proportions. Computational Statistics \& Data Análisis 2004; 45: 449-456. DOI : 10.1016/ S0167-9473(03)00007-0.

19. Cohen LA, Kendall ME, Zang E, Meschter C, Rose DP. Modulation of NNitrosomethylurea-Induced Mammary Tumor Promotion by Dietary Fiber and Fat. Journal of National Cancer Institution 1991; 83: 496-501. DOI: 10.1093/jnci/83.7.496.

20. Schaarschmidt F, Sill M, Hothorn LA. Approximate Simultaneous Confidence Intervals for Multiple Contrasts of Binomial Proportions. Biometrical Journal 2008; 50: 782-792. DOI: 10.1002/bimj.200710465.

21. Newcombe RG. Estimating the difference between differences: measurement of additive scale interaction for proportions. Statistics in Medicine 2001; 20: 2801-2994. DOI: 10.1002/sim.925.

22. Zou G, Huang W, Zhang X. A note on confidence interval estimation for a linear function of binomial proportions. Computational Statistics \& Data Análisis 2009 ; 53: 1080-1085. DOI: $10.1016 /$ j.csda.2008.09.033.

23. Beal SL. Asymptotic Confidence Intervals for the Difference between Two Binomial Parameters for Use with Small Samples. Biometrics 1987; 43: 941-950.

24. Mee RW. Confidence Bounds for the difference between two probabilities. Biometrics 1984; 40: 1175-1176.

25. Koopman PAR. Confidence intervals for the ratio of two binomial proportions. Biometrics 1984; 40: 513-517.

26. Nam JM. Confidence limits for the ratio of two binomial proportions based on likelihood scores: Non-iterative method. Biometrical Journal 1995; 37: 375-379. DOI: 10.1002/bimj. 
4710370311.

27. Gart JJ, Nam J. Approximate interval estimation of the ratio of binomial parameters: A review and corrections for skewness. Biometrics 1988; 44: 323-338.

28. Martín Andrés A, Herranz Tejedor I. Propiedades del estadístico $z$ en el contexto del test de equivalencia de dos proporciones. XI Conferencia Española de Biometría y I Encuentro Iberoamericano de Biometría (Salamanca, Spain) 2007: 79-80.

29. Barnard GA. Significance tests for $2 \times 2$ tables. Biometrika 1947; 34: 123-138.

30. Röhmel J, Mansmann U. Unconditional non-asymptotic one-sided tests for independent binomial proportions when the interest lies in showing non-inferiority and/or superiority. Biometrical Journal 1999; 41: 149-170. DOI: 10.1002/(SICI)15214036(199905).

31. Martín Andrés A, Herranz Tejedor I. Exact unconditional non-classical tests on the difference of two proportions. Computational Statistics and Data Analysis 2004; 45 : 373-388. DOI: 10.1016/S0167-9473(02)00351-1.

32. Röhmel J, Mansmann U. Exact tests of equivalence and efficacy with a non-zero lower bound for comparative studies by I.S.F. Chan (Letters to the Editor). Statistics in Medicine 1999; 18: 1734-1737. DOI: 10.1002/(SICI)1097-0258(19990715). 
Table 1: Diet and tumor study

\begin{tabular}{lcccc}
\hline & \multicolumn{2}{c}{ Fiber } & \multicolumn{2}{c}{ No Fiber } \\
\cline { 2 - 5 } & High Fat & Low Fat & High Fat & Low Fat \\
\hline Sample size $\left(n_{i}\right)$ & 30 & 30 & 30 & 30 \\
Rats showing cancer $\left(x_{i}\right)$ & 20 & 14 & 27 & 19 \\
\hline Effect & $\beta_{1}$ & $\beta_{2}$ & $\beta_{3}$ & $\beta_{4}$ \\
\hline$L_{1}=$ Fiber $\times$ Fat & +1 & -1 & -1 & +1 \\
$L_{2}=$ Fiber & +1 & +1 & -1 & -1 \\
$L_{3}=$ Fat & +1 & -1 & +1 & -1 \\
\hline
\end{tabular}

Table 2: Multicenter clinical trial data

\begin{tabular}{lccc}
\hline Location & Sample size $\left(n_{i}\right)$ & Fever cases $\left(x_{i}\right)$ & Coefficients $\left(\beta_{i}\right)$ \\
\hline Bangladesh & 158 & 73 & $158 / 675$ \\
Brazil & 107 & 32 & $107 / 675$ \\
India & 175 & 44 & $175 / 675$ \\
Peru & 92 & 34 & $92 / 675$ \\
Vietnam & 143 & 104 & $143 / 675$ \\
Total & 675 & 287 & 1 \\
\hline
\end{tabular}


Table 3: Analysis of the data in Tables 1 and 2

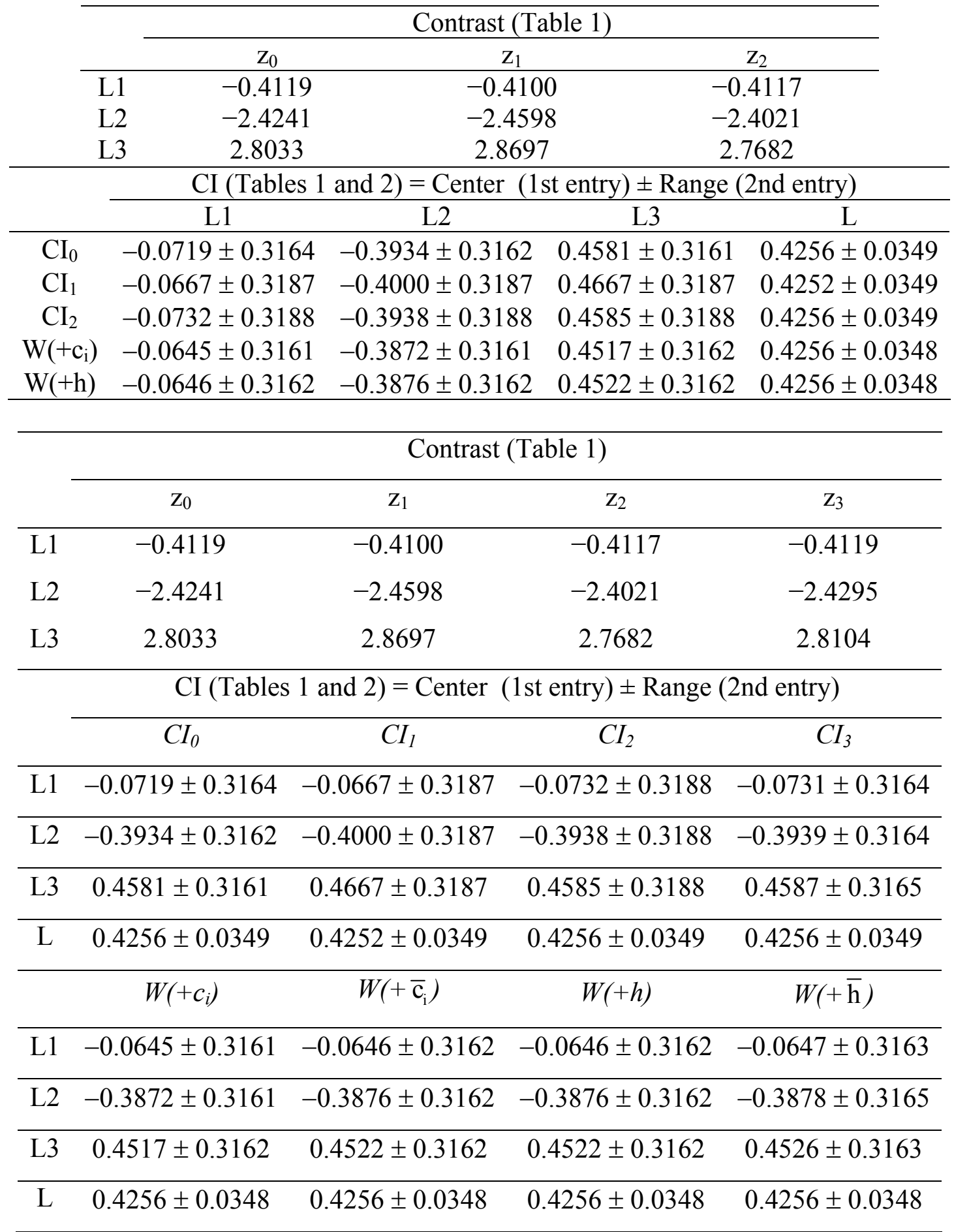


Table 4: Exact coverage $(R)$ and width $(W)$ of $S\left(\right.$ score), W(+2/K) (Price and Bonett) and $W\left(+h_{i}^{\prime}\right)($ new) intervals for a confidence $=95 \%$ $\mathrm{K}=3$

\begin{tabular}{|c|c|c|c|c|c|c|c|c|c|c|c|c|}
\hline \multirow{2}{*}{$\begin{array}{l}\text { Method: } \\
n_{1} / n_{2} / n_{3} \\
\end{array}$} & \multicolumn{4}{|c|}{$\mathrm{W}(+2 / K)$} & \multicolumn{4}{|c|}{$\mathrm{W}\left(+h_{i}^{\prime}\right)$} & \multicolumn{4}{|c|}{$\mathrm{S}$} \\
\hline & Rmean & Rmin & Wmean & Rbelow93 & Rmean & Rmin & Wmean & Rbelow93 & Rmean & Rmin & Wmean & Rbelow93 \\
\hline \multicolumn{13}{|l|}{$\beta_{i}=(1 / 3,1 / 3,1 / 3)$} \\
\hline $30 / 30 / 30$ & 95.2 & 92.7 & 0.16 & 0.0 & 95.6 & 93.1 & 0.17 & 0.0 & 94.8 & 92.9 & 0.16 & 0.0 \\
\hline $30 / 10 / 10$ & 95.5 & 92.2 & 0.25 & 0.1 & 96.7 & 93.5 & 0.26 & 0.0 & 95.0 & 90.2 & 0.24 & 0.0 \\
\hline \multicolumn{13}{|l|}{$\beta_{i}=(1,-1 / 2,-1 / 2)$} \\
\hline $10 / 10 / 10$ & 95.5 & 87.6 & 0.58 & 0.6 & 96.9 & 93.1 & 0.64 & 0.0 & 95.1 & 92.4 & 0.58 & 0.1 \\
\hline $30 / 30 / 30$ & 95.2 & 92.2 & 0.35 & 0.0 & 95.6 & 93.0 & 0.36 & 0.0 & 94.9 & 93.9 & 0.35 & 0.0 \\
\hline $30 / 10 / 10$ & 95.4 & 92.1 & 0.44 & 0.1 & 96.5 & 92.5 & 0.47 & 0.0 & 94.4 & 92.4 & 0.44 & 0.3 \\
\hline $30 / 30 / 30$ & 95.1 & 93.0 & 0.65 & 0.0 & 95.6 & 94.1 & 0.66 & 0.0 & 95.1 & 94.4 & 0.64 & 0.0 \\
\hline $30 / 10 / 10$ & 95.4 & 89.4 & 1.02 & 4.7 & 96.7 & 91.5 & 1.09 & 0.6 & 95.5 & 89.8 & 0.99 & 0.1 \\
\hline $30 / 20 / 10$ & 95.3 & 89.7 & 1.00 & 8.2 & 96.6 & 91.0 & 1.07 & 3.3 & 95.5 & 88.9 & 0.97 & 0.0 \\
\hline \multicolumn{13}{|l|}{$\beta_{i}=(1,1,-1)$} \\
\hline $10 / 10 / 10$ & 95.6 & 90.0 & 0.83 & 0.2 & 97.0 & 90.5 & 0.90 & 0.0 & 94.3 & 92.1 & 0.82 & 7.0 \\
\hline $30 / 30 / 30$ & 95.2 & 92.5 & 0.49 & 0.0 & 95.7 & 92.8 & 0.51 & 0.0 & 94.8 & 92.4 & 0.49 & 0.0 \\
\hline $30 / 10 / 10$ & 95.5 & 90.1 & 0.73 & 0.0 & 96.8 & 92.6 & 0.79 & 0.0 & 95.0 & 91.8 & 0.73 & 0.0 \\
\hline $30 / 20 / 10$ & 95.4 & 90.7 & 0.66 & 0.1 & 96.4 & 93.4 & 0.69 & 0.0 & 95.1 & 93.0 & 0.65 & 0.0 \\
\hline
\end{tabular}


Table 4 (cont.): $K=4$

\begin{tabular}{|c|c|c|c|c|c|c|c|c|c|c|c|c|}
\hline \multirow{2}{*}{\begin{tabular}{|l|} 
Method: \\
$n_{1} / n_{2} / n_{3} / n_{4}$ \\
\end{tabular}} & \multicolumn{4}{|c|}{$\mathrm{W}(+2 / K)$} & \multicolumn{4}{|c|}{$\mathrm{W}\left(+h_{i}^{\prime}\right)$} & \multicolumn{4}{|c|}{$\mathrm{S}$} \\
\hline & Rmean & Rmin & Wmean & Rbelow93 & Rmean & Rmin & Wmean & Rbelow93 & Rmean & Rmin & Wmean & Rbelow93 \\
\hline \multicolumn{13}{|l|}{$\beta_{I}=(1 / 4,1 / 4,1 / 4,1 / 4)$} \\
\hline $10 / 10 / 10 / 10$ & 95.3 & 92.9 & 0.24 & 0.0 & 97.2 & 93.6 & 0.27 & 0.0 & 93.8 & 91.7 & 0.24 & 7.1 \\
\hline $20 / 20 / 20 / 20$ & 95.1 & 93.2 & 0.17 & 0.0 & 96.0 & 93.8 & 0.18 & 0.0 & 94.5 & 92.9 & 0.17 & 0.0 \\
\hline $20 / 20 / 10 / 10$ & 95.2 & 93.0 & 0.21 & 0.0 & 96.8 & 94.0 & 0.23 & 0.0 & 94.4 & 93.1 & 0.21 & 0.0 \\
\hline $20 / 15 / 10 / 5$ & 95.3 & 85.7 & 0.24 & 0.6 & 97.5 & 93.7 & 0.27 & 0.0 & 95.1 & 93.1 & 0.24 & 0.0 \\
\hline \multicolumn{13}{|l|}{$\beta_{i}=(-1,1,-1,1)$} \\
\hline $10 / 10 / 10 / 10$ & 95.3 & 93.0 & 0.96 & 0.0 & 97.1 & 93.6 & 1.06 & 0.0 & 93.8 & 92.0 & 0.94 & 6.4 \\
\hline $20 / 20 / 20 / 20$ & 95.1 & 93.6 & 0.69 & 0.0 & 96.1 & 93.8 & 0.73 & 0.0 & 94.5 & 92.1 & 0.69 & 0.0 \\
\hline $20 / 20 / 10 / 10$ & 95.2 & 92.7 & 0.84 & 0.0 & 96.7 & 94.2 & 0.91 & 0.0 & 94.4 & 93.0 & 0.83 & 0.0 \\
\hline $20 / 15 / 10 / 5$ & 95.3 & 91.3 & 0.97 & 0.5 & 97.5 & 94.0 & 1.08 & 0.0 & 95.1 & 92.9 & 0.96 & 0.0 \\
\hline \multicolumn{13}{|l|}{$\beta_{I}=(1 / 3,1 / 3,1 / 3,1)$} \\
\hline $10 / 10 / 10 / 10$ & 94.9 & 90.2 & 0.55 & 4.5 & 96.9 & 92.6 & 0.60 & 0.0 & 95.3 & 92.8 & 0.54 & 0.0 \\
\hline $20 / 20 / 20 / 20$ & 94.9 & 92.3 & 0.40 & 0.1 & 95.9 & 93.7 & 0.41 & 0.0 & 95.2 & 94.3 & 0.39 & 0.0 \\
\hline $20 / 20 / 10 / 10$ & 94.8 & 87.0 & 0.53 & 13.8 & 96.7 & 92.6 & 0.57 & 0.4 & 95.4 & 93.5 & 0.52 & 0.0 \\
\hline $20 / 15 / 10 / 5$ & 94.8 & 80.8 & 0.69 & 40.7 & 97.8 & 93.5 & 0.77 & 0.0 & 95.6 & 90.3 & 0.65 & 0.1 \\
\hline \multicolumn{13}{|l|}{$\beta_{i}=(-3,-1,1,3)$} \\
\hline $10 / 10 / 10 / 10$ & 95.1 & 91.4 & 2.14 & 0.1 & 97.0 & 93.6 & 2.35 & 0.0 & 95.0 & 93.2 & 2.12 & 0.0 \\
\hline $20 / 20 / 20 / 20$ & 95.0 & 92.9 & 1.54 & 0.0 & 95.9 & 94.0 & 1.61 & 0.0 & 94.7 & 93.8 & 1.53 & 0.0 \\
\hline $20 / 20 / 10 / 10$ & 95.0 & 90.1 & 1.86 & 0.6 & 96.6 & 91.8 & 2.01 & 0.0 & 95.2 & 93.9 & 1.85 & 0.0 \\
\hline $20 / 15 / 10 / 5$ & 95.1 & 85.8 & 2.29 & 35.0 & 97.7 & 94.0 & 2.55 & 0.0 & 95.6 & 92.3 & 2.23 & 0.0 \\
\hline
\end{tabular}

